\title{
Deep Learning Based Covid-19 Detection With A Novel CT Images Dataset: EFSCH-19
}

\author{
Oğuzhan Katar $^{1 *}$, Erkan Duman ${ }^{2}$ \\ 1* Firat University, Faculty of Technology, Departmant of Software Engineering, Elazig, Turkey, (ORCID: 0000-0002-5628-3543), okatar@ firat.edu.tr \\ ${ }^{2}$ Firat University, Faculty of Engineering, Departmant of Computer Engineering, Elazig, Turkey, (ORCID: 0000-0003-2439-7244), erkanduman@ firat.edu.tr
}

(International Symposium on Multidisciplinary Studies and Innovative Technologies (ISMSIT) 2021 - 21-23 October 2021)

(DOI: 10.31590/ejosat.1021030)

ATIF/REFERENCE: Katar, O., \& Duman, E. (2021). Deep Learning Based Covid-19 Detection With A Novel CT Images Dataset: EFSCH-19. European Journal of Science and Technology, (29), 150-155.

\begin{abstract}
COVID-19 pandemic has negatively affected the whole world in many ways. Since its inception, various methods and approaches have been developed. The common feature of these solution searches is minimizing the social and economic damages of the COVID19 pandemic. In this article, we developed our deep learning-based model for the detection of COVID-19 disease from chest CT images. However, we did not use the publicly available datasets used in most studies in the literature. Because, in public datasets; there are problems such as low samples, incorrectly labeled images and unbalanced distribution. Due to such problems, we thought that our model would not reach the desired high accuracy values. We used our dataset, which has not been included in any deep learning study before, from Elazig Fethi Sekin City Hospital, for the first time in the training of our model. Our model was trained with 800 positive and 800 normal chest CT images and then tested with 400 randomly selected test images. As a result of these tests, accuracy rate of $\% 97.5$ was achieved. When the results of our study are evaluated, it is thought that it can help physicians in the detection of COVID-19 disease.
\end{abstract}

Keywords: COVID-19, Deep learning, Image processing, CT scans, CNN, Image Classification, Diagnosing

\section{Yeni Bir CT Görüntüleri Veri Kümesi ile Derin Öğrenme Tabanlı Covid-19 Tespiti: EFSCH-19}

$\ddot{O} \mathbf{z}$

COVID-19 pandemisi tüm dünyayı birçok yönden olumsuz etkiledi. Kurulduğu günden bu yana çeşitli yöntem ve yaklaşımlar geliştirilmiştir. Bu çözüm arayışlarının ortak özelliği, COVID-19 pandemisinin sosyal ve ekonomik zararlarını en aza indirmektir. Bu çalışmada, göğüs BT görüntülerinden COVID-19 hastalığının tespiti için derin öğrenme tabanlı modelimizi geliştirdik. Ancak literatürdeki çoğu çalışmada kullanılan halka açık veri setlerini kullanmadık. Çünkü halka açık veri setlerinde; düşük sayıda eleman, yanlış etiketlenmiş görüntüler ve dengesiz dağılım gibi sorunlar mevcut. Bu tür problemlerden dolayı modelimizin istenilen yüksek doğruluk değerlerine ulaşamayacağını düşündük. Elazığ Fethi Sekin Şehir Hastanesi'nden daha önce herhangi bir derin öğrenme çalışmasına dahil edilmemiş veri setimizi modelimizin eğitiminde ilk kez kullandık. Modelimiz 800 pozitif ve 800 normal göğüs BT görüntüsü ile eğitildi ve ardından rastgele seçilmiş 400 test görüntüsü ile test edildi. Bu testler sonucunda \%97,5 doğruluk oranına ulaşılmıştır. Çalışmamızın sonuçları değerlendirildiğinde hekimlere COVID-19 hastalığının tespitinde yardımcı olabileceği düşünülmektedir.

Anahtar Kelimeler: COVID-19, Derin öğrenme, Görüntü işleme, BT taramaları, ESA, Görüntü sınıflandırma, Teşhis.

\footnotetext{
* Corresponding Author: okatar@firat.edu.tr
} 


\section{Introduction}

Our world has dealt with many epidemics from the past to the present and is currently struggling with the 'COVID-19' global epidemic that emerged in December 2019 in Wuhan, China [1]. This is a respiratory disease caused by SARS-CoV2. The most prominent symptoms of COVID-19 include high fever, dry cough, respiratory problems, severe sore throat and diarrhea. In addition to the stated findings, loss of taste and smell sense, joint pain and nasal congestion are also observed in most patients [2]. There is no measure or vaccine available to completely protect against new types of infectious diseases such as COVID-19. As a result, it can be fairly quick and easy for an infected person to spread the disease to other people. Establishing an isolated area between healthy and infected people and promoting the use of masks are ways to control the spread of virus infection [3].

RT-PCR (Reverse-Transcriptase Polymerase Chain Reaction) test is applied for the diagnosis of COVID-19 [4]. This test detects the presence of the virus by observing the RNA of the COVID-19 virus and ensures its identification. In order to perform the test, a swab should be taken from the throat and nose with the help of a cotton swab. The low sensitivity, timeconsuming testing, and the need for special tools that are not available in standard laboratories main disadvantages of RT-PCR are. RT-PCR creates difficulties in detecting infected people and preventing the spread of the disease, due to the fact that some countries do not have enough test kits and the mentioned disadvantages. As an alternative to these difficulties, radiological imaging techniques are used for the diagnosis of COVID-19 [5].

$\mathrm{X}$-Ray-based radiological imaging modalities such as chest radiograph and chest $\mathrm{CT}$ scan can be helpful to isolate people whose lungs are infected and to initiate treatment early [6]. These methods can simply reveal the visual radiological features of COVID-19, especially the ground-glass finding. Radiologists mostly prefer chest radiograph to detect the disease, because $\mathrm{X}$ ray equipment is available in most hospitals. Chest radiograph taken with the help of X-ray devices are insufficient, especially when the soft tissues of the organs are desired to be observed. To overcome this deficiency, CT scanning, which has the ability to successfully observe the soft tissues of the organs, is used [8]. Radiologists should refer to CT images in the analysis of the ground glass areas on the soft tissues. In this pandemic period, which affects the health sector very badly, many radiologists should undertake duties in the diagnosis of COVID-19 disease. However, the report generation process is a time-consuming and error-prone task due to human factors. For these reasons, computers should be used to detect the disease from radiological images. 'Deep Learning' methods, which is the subtitle of artificial intelligence, are used by researchers in the classification and analysis of images. Especially in the studies of disease detection from medical images, the number of elements of the dataset is of great importance. Because when the number of elements in the dataset is low, it is more efficient to use pretrained neural networks, while it is more appropriate to train our own model when the number of elements of the dataset increases.

The main purpose of this article study is to classify CT images with COVID-19 (+) and normal findings with high accuracy rates using our model in Convolutional Neural Network architecture. During the training phase of the specified model, computerized tomography images of real patients submitted to us were used, thanks to the administrative permissions obtained by the TR Ministry of Health and the Chief Physician of Elazig Fethi Sekin City Hospital. Our dataset consists of 1000 COVID-19 positive and 1000 COVID-19 negative computed tomography images. In our dataset, it was not sufficient to have only ground glass areas in the images that were positive for COVID-19, and the condition that the diagnosis of "U07.3" ICD10 was entered by the specialist doctor was strictly observed. As a result of our study, the performance results of our model were shared, and a fast and reliable method was proposed for the detection of COVID-19 disease from computed tomography images.

The rest of this paper is organized as follows. Section 2 includes other studies in the literature on deep learning techniques for image classification. The details of the dataset, model and performance metrics is described in Section 3. The analysis of test results and experimental results of our COVID19 classifier model are given in Section 4. Conclusion part of the study is in Section 5.

\section{Related Works}

In this section, some studies from X-ray-based radiological images in the literature on COVID-19 detection are examined. The main idea behind these studies is to classify the covid infection in the lungs as positive or negative based on the findings in the radiological images.

In the study of [7], researchers conducted a deep learningbased study that could be useful in detecting COVID-19 from chest CT images. They used transfer learning models to learn and extract important feature vectors. Due to the insufficient number of elements in the publicly available CT datasets, they created a balanced dataset combined from three different sources. The dataset, which consists of 11,209 images in total, contains 5,602 positive and 5,607 negative samples. They used the pre-trained VGG16, DenseNet121, InceptionV3, Inception-Resnet, Xception and the CNN architecture model they developed in order to classify the images. VGG16 reached the highest accuracy with \%98.7 among all transfer learning models.

In the study of [8], COVID-19 detection was presented using XGBoost in the classification phase of convolutional neural network $(\mathrm{CNN})$ in feature extraction from chest $\mathrm{CT}$ scan images. They created the dataset from a total of $708 \mathrm{CT}$ images, 312 of which were infected with COVID-19 and 396 with normal findings. Test data classified with the XGBoost model as a result, 95.07 accuracy, 95.09 recall, 94.99 precision, 95 F-score and 95 AUC values were obtained on the findings in the radiological images.

In the study of [9], researchers developed the model called COVNet(COVID-19 detection neural network) for COVID19 detection. This model, which can classify on CT images, was trained on 4352 images taken from 3222 patients. The accuracy obtained from COVNet was \%95. Researchers could not publicly publish the dataset.

In the study of [10], a diagnostic system was developed using CT images to help doctors diagnose COVID-19 patients. Unlike two-dimensional studies, 3D deep learning models based on CT images were also applied for COVID-19 detection [11]. Several works have also applied for COVID19 infection regions 
segmentation. In these studies, VB-Net and UNet++ based models were used [12].

In the study of [13], they used UNet++ to extract segmented lesions from CT images and predict COVID-19 and other viral pneumonia types. Thanks to the proposed model, the reading time of radiologists was shortened by $65 \%$.

In the study of [14], they proposed a deep neural network that uses synthetic images to detect COVID-19. An accuracy rate of $98.7 \%$ was obtained from the proposed model.

\section{Material and Method}

\subsection{Dataset}

The dataset used in this study was composed of two classes called COVID-19 (+) and COVID-19 (-). There are 1000 chest CT images in each class, so our dataset is in a balanced structure. Real patient images taken with Computed Tomography devices in Elazig Fethi Sekin City Hospital are the source of our dataset elements. There are an average of 70 frames in a chest CT scan taken at Elazig Fethi Sekin City Hospital. An exemplary chest CT sequence is shown in Figure 1, with patient confidentiality in mind.

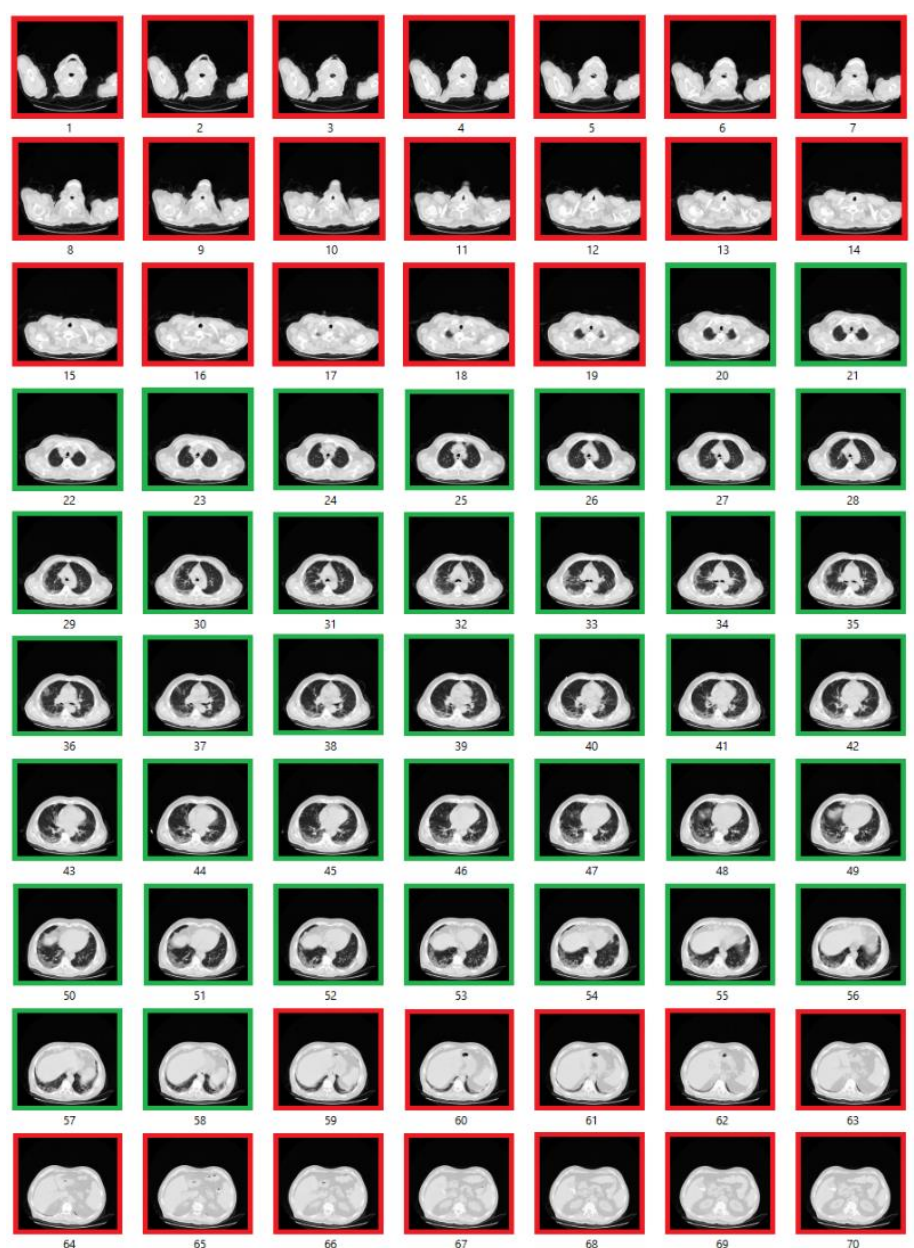

Figure 1. Chest CT Scan

In Figure 1, frames with useful information that need to be included in the process in order to detect COVID-19 are indicated with a green, and frames without useful information are indicated with a red. Chest radiograph of the same patient is shown in Figure 2. with the same coloring method. These areas correspond to areas containing useful information about the patient's chest CT image.

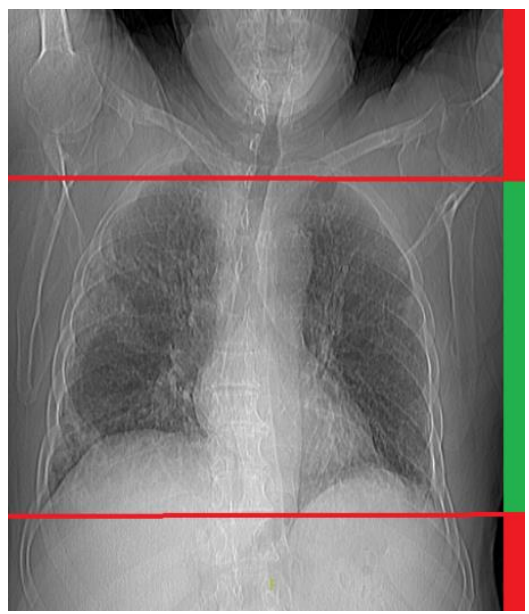

Figure 2. Chest Radiograph

The elements in the dataset have a size of $768 \times 768$ px. This size is equivalent to its raw form in the PACS server. The images taken by the CT device are simultaneously transferred to the PACS server in DICOM format with the help of integration. In our study, instead of using DICOM format, "JPG" conversion was performed by using the conversion features offered by the PACS application. Figure 3. shows a sample of CT scans of COVID-19 (+) and COVID-19 (-) patients from the dataset.
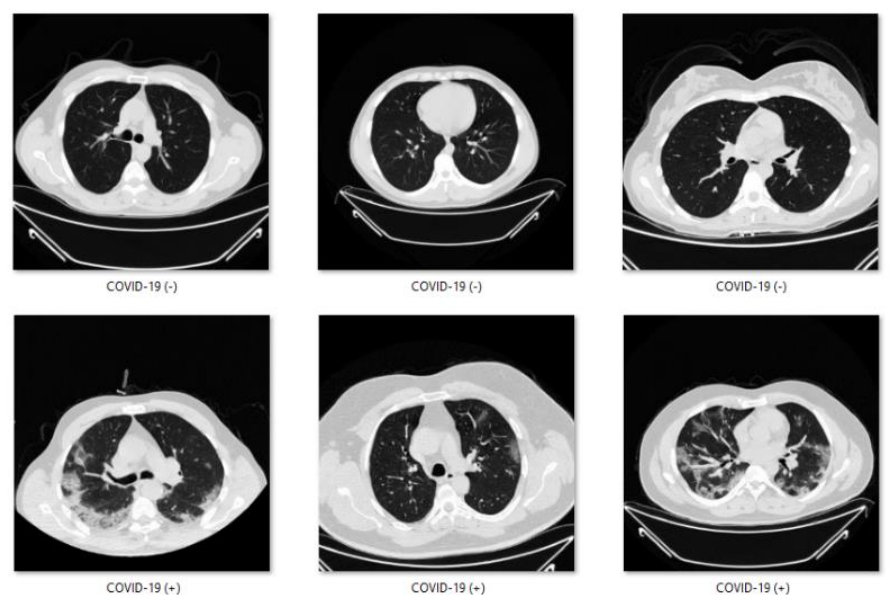

Figure 3. Sample of CT Scans

According to the CO-RADS study [15]; CT image findings for COVID-19 detection are divided into 7 categories. These categories are named sequentially from CO-RADS-0 to CORADS-6. Brief descriptions of each category are as follows.

- CO-RADS-0 is chosen for situations where CT scans are incomplete or of poor quality

- CO-RADS-1 means very low suspicion for lung involvement by COVID-19.

- CO-RADS-2 means a low level of suspicion based on CT findings considered incompatible with COVID-19.

- CO-RADS-3 refers to suspicious findings for pulmonary involvement of COVID-19.

- CO-RADS-4 refers to a highly suspected condition typical of COVID-19 but also somewhat similar to other pneumonia findings.

- CO-RADS-5 means very high suspicion for lung involvement by COVID-19

- CO-RADS-6 is used to indicate COVID-19 proven by RT-PCR test results. 
In our dataset; Images with the COVID-19 (+) label consist of CO-RADS-4 and CO-RADS-5, and those with the COVID-19 (-) label consist of CO-RADS-1, according to the CO-RADS classification. 3-dimensional t-SNE plot of the dataset is given in Figure 4.

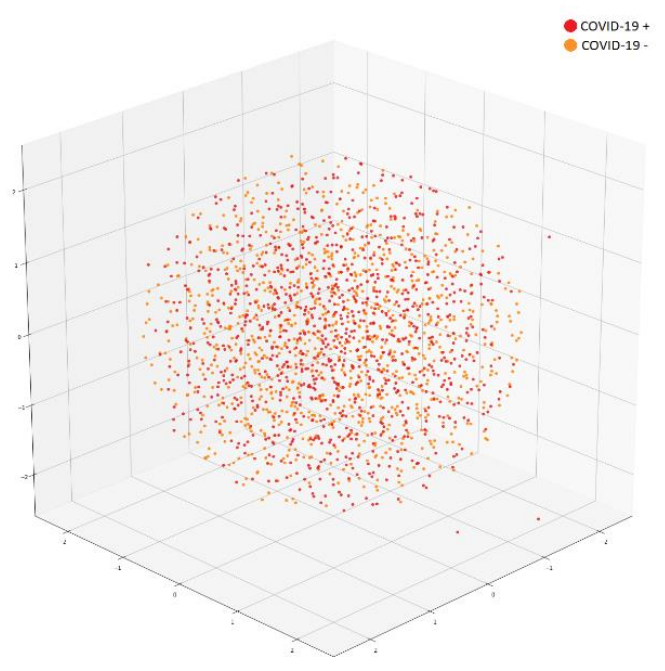

Figure 4. 3D t-SNE Plot of Dataset

\subsection{Model Training}

Before starting the training of our model, we resized the $768 \times 768$ px images in our dataset to $150 \times 150$ px. Then, we divided the images we have into \%80 train and \%20 test. The dataset sample distribution used in model training and testing stages is shown in Table I.

Table 1. Number of Images in Dataset

\begin{tabular}{l|c|c|c}
\hline & COVID-19 (+) & COVID-19 (-) & Total \\
\hline Train & 800 & 800 & 1600 \\
\hline Test & 200 & 200 & 400 \\
\hline Total & 1000 & 1000 & 2000 \\
\hline
\end{tabular}

In the training of our model, Python was preferred as the programming language and the Keras library was used. Epoch number: 50 , batch size: 64 , learning rate: 0.01 , adam optimizer function and binary cross entropy loss hyperparameters were preferred.

\subsection{Proposed Model}

The CNN model we developed for the solution of the problem consists of 19 layers as shown in Figure 5. Our model accepts the input image as $150 \times 150 \mathrm{px}$ and transmits it to the convolution layer. The kernel size of the convolution layers in the second, sixth and tenth layers is equal to $3 \times 3$. A pooling layer is used to extract feature maps from the image after each convolution layer. However, before each pooling layer, an activation layer (ReLu) were used to make our network more organized and optimized during training. The main reason for using the pooling layer in our model is that it reduces the input size for the next convolution layer. We've set 'Dropout Rate' to $\mathrm{p}=0.5$, which allows for maximum regulation. Otherwise, if all the weights are learned together in the networks, normally some links will gain more predictive ability than others. Dropout is also used in the Fully Connected Layer so that our model can avoid over-learning. Sigmoid activation function, which is very effective in binary classification, is used in the classifier layer of our model. In the output layer, our result is classified as 0 or 1 .

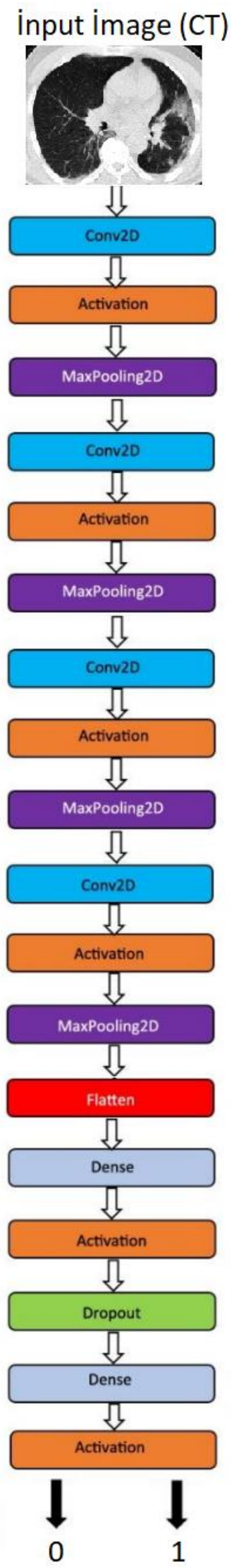

Figure 5. Proposed Model's Details 


\subsection{Performance Metrics}

In this study, the classification performance of the COVID19 classification model proposed is evaluated with confusion matrix-based performance measures. The confusion matrix provides information about the relationship between the image tags that the model predicts in the output layer and the actual image tags. The metrics used to demonstrate this relationship are accuracy, precision, specificity, recall and F-1.

The main purpose of performing the binary classification model is to determine whether there is a COVID-19 infection in the lungs of the patient. In binary classification models, the predictions can only be 0 or 1 . These estimates may match the patient's actual condition or may be inaccurate. Considering the specified structure, four different situations can occur. The first case is called True Positive (TP) and involves predicting an image with the COVID-19 (+) tag as COVID-19 (+) by the model. The second case is called False Positive (FP) and involves the model predicting an image with the COVID19 (-) tag as COVID-19 (+). The third case is called True Negative (TN) and involves the model predicting an image with the COVID-19 (-) tag as COVID-19 (-). The fourth case is called False Negative (FN) and involves the model predicting an image with the COVID-19 (+) tag as COVID-19 (-). A successful model is expected to have high TP and TN rates. The following equations of metrics are used to validate the performance of the outputs predicted by our model.

Accuracy $\left(A_{c}\right)$ is defined as the division of all correct classifications by the total number of classifications. How to calculate $\left(A_{c}\right)$ is shown in (1).

$$
\mathrm{A}_{\mathrm{c}}=\frac{\mathrm{TP}+\mathrm{TN}}{\mathrm{TP}+\mathrm{TN}+\mathrm{FP}+\mathrm{FN}}
$$

Precision $\left(\mathrm{P}_{\mathrm{r}}\right)$ shows how many of the values we estimated as positive are actually positive. A model with a low Precision $\left(\mathrm{P}_{\mathrm{r}}\right)$ value makes a large number of FP estimates. The mathematical equation necessary to calculate this value is given in (2).

$$
\mathrm{P}_{\mathrm{r}}=\frac{\mathrm{TP}}{\mathrm{TP}+\mathrm{FP}}
$$

Specificity $\left(\mathrm{S}_{\mathrm{p}}\right)$ shows how many of the values we estimated as negative are actually negative. The equation used to calculate the Specificity $\left(\mathrm{S}_{\mathrm{p}}\right)$ is shown in (3).

$$
\mathrm{S}_{\mathrm{p}}=\frac{\mathrm{TN}}{\mathrm{TN}+\mathrm{FP}}
$$

Recall $\left(R_{c}\right)$ is the ratio of the number of correctly classified positive samples to the total number of positive samples. The equation used to calculate the Recall $\left(R_{c}\right)$ is shown in (4).

$$
\mathrm{R}_{\mathrm{c}}=\frac{\mathrm{TP}}{\mathrm{TP}+\mathrm{FN}}
$$

F-score $\left(\mathrm{F}_{1}\right)$ value is the harmonic average of Precision $\left(\mathrm{P}_{\mathrm{r}}\right)$ and Recall $\left(R_{c}\right)$ values. It is one of the most important success criteria when the dataset distribution is unbalanced. The calculation of the F-score $\left(\mathrm{F}_{1}\right)$ value is shown in (5).

$$
\mathrm{F}_{1}=2 * \frac{\mathrm{P}_{\mathrm{r}} * \mathrm{R}_{\mathrm{c}}}{\mathrm{P}_{\mathrm{r}}+\mathrm{R}_{\mathrm{c}}}
$$

\section{Experimental Results}

The proposed model for detecting COVID-19 infection from CT images was trained in the Google Colab environment. As a result of 50 epochs, each lasting $45 \mathrm{~ms}$, our model reached accuracy rate of \%97.5. The graphic representation of the accuracy values of the training phase is shown in Figure 6.

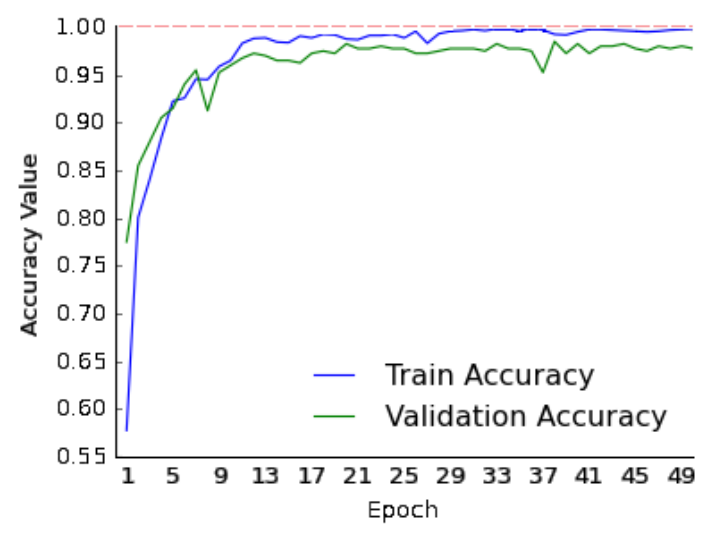

Figure 6. Train and Validation Accuracy of Model

As seen in Figure 6, train accuracy and validation accuracy did not reach ' 1.00 ' at any epoch. The reason for this is the effective use of the "Dropout" layer. The display of "Loss" values, which is another necessary graphic for the interpretation of the training phase, is given in Figure 7.

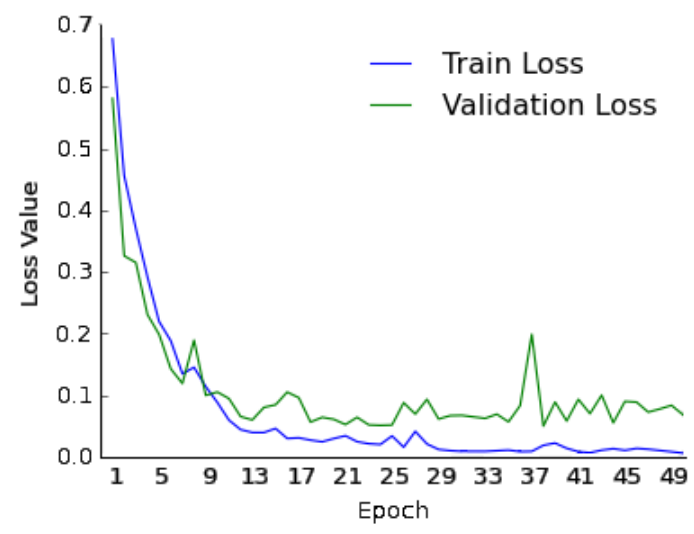

Figure 7. Train and Validation Loss of Model

Figure 8 shows the confusion matrix and other performance metrics of our model. The accuracy rate given as $\% 97.5$ by the console output as a result of the training phase can be proven from Figure 8. The closer these values are to 1, the higher the success rate and discrimination ability of our model.

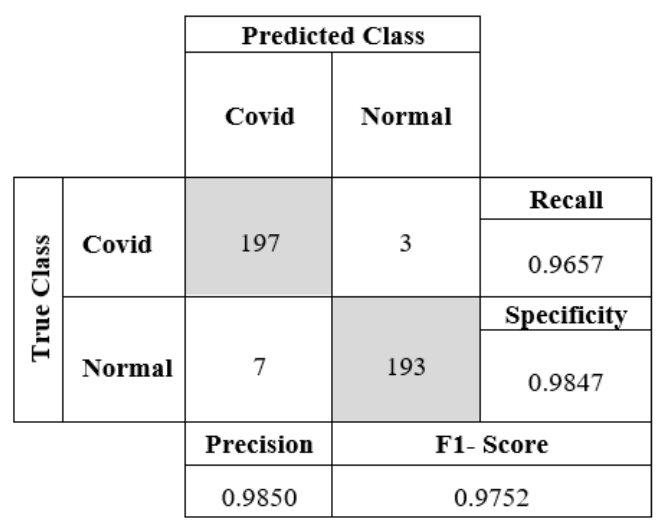

Figure 8. Confusion Matrix of Model's Outputs 
It is very important that margin of error due to misclassification is close to zero in artificial intelligence models designed to be used in the detection of diseases such as COVID19 that can directly affect human life. Increasing the number of elements in the dataset is among the primary solutions in order to minimize the specified margins of error. Because the more elements the dataset has, the more comprehensive it will be. In this way, deep learning models will dominate various types of examples. Figure 9 shows random samples of chest CT images reserved for testing before the training phase started, which were successfully classified by our model.
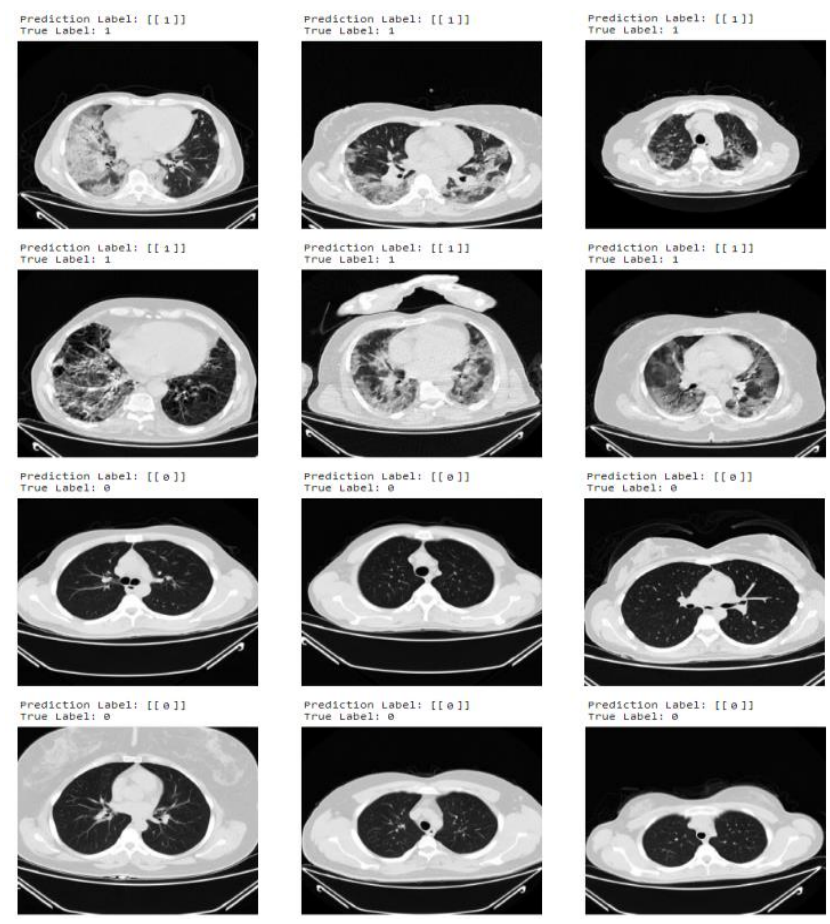

Figure 9. Successfully Classified CT Images Samples

\section{Conclusion}

In this study, we propose an uncomplicated and useful CNN model for the detection of COVID-19 from CT images. Our model is trained to classify CT images of patients with and without COVID19 symptoms. The dataset we used was created specifically for this study. Although high values were achieved in all performance metrics, the predictive ability of the model could be taken to the next level if more images were included in the training phase. In this study, it is aimed to present a deep learning-based fast and reliable decision support system to radiologists who have been trying to detect COVID-19. Today the technological devices used in the field of imaging are in a structure that can work integrated with the method we have developed for the detection of the disease. Designing and implementing such decision support systems can improve the future of the healthcare industry. The availability of similar studies is an important advantage in the fight against emergencies such as the COVID-19 epidemic, which spreads rapidly and information about the disease is scarce.

\section{References}

Wang, D., Hu, B., Hu, C., Zhu, F., Liu, X., Zhang, J., ... \& Peng, Z. (2020). Clinical characteristics of 138 hospitalized patients with 2019 novel coronavirus-infected pneumonia in Wuhan, China. Jama, 323(11), 1061-1069.

e-ISSN: 2148-2683
Singhal, T. (2020). Uma revisão da doença de Coronavírus-2019 (COVID-19). Indian J Pediatr, 87, 281-286.

Chan, J. F., Lau, S. K., To, K. K., Cheng, V. C., Woo, P. C., \& Yuen, K. Y. (2015). Middle East respiratory syndrome coronavirus: another zoonotic betacoronavirus causing SARS-like disease. Clinical microbiology reviews, 28(2), 465-522.

Wang, W., Xu, Y., Gao, R., Lu, R., Han, K., Wu, G., \& Tan, W. (2020). Detection of SARS-CoV-2 in different types of clinical specimens. Jama, 323(18), 1843-1844.

Li, L., Qin, L., Xu, Z., Yin, Y., Wang, X., Kong, B., ... \& Xia, J. (2020). Artificial intelligence distinguishes COVID-19 from community acquired pneumonia on chest CT. Radiology.

Singh, D., Kumar, V., \& Kaur, M. (2020). Classification of COVID-19 patients from chest CT images using multiobjective differential evolution-based convolutional neural networks. European Journal of Clinical Microbiology \& Infectious Diseases, 39(7), 1379-1389.

Jashnani, K., Nargunde, R., Shah, Y., \& Raul, N. (2021, June). COVID-19 Prediction from CT Scans using Deep-Learning. In 2021 International Conference on Communication information and Computing Technology (ICCICT) (pp. 1-6). IEEE.

Carvalho, E. D., Carvalho, E. D., de Carvalho Filho, A. O., De Araújo, F. H. D., \& Rabêlo, R. D. A. L. (2020, July). Diagnosis of COVID-19 in CT image using CNN and XGBoost. In 2020 IEEE Symposium on Computers and Communications (ISCC) (pp. 1-6). IEEE.

Li, L., Qin, L., Xu, Z., Yin, Y., Wang, X., Kong, B., ... \& Xia, J. (2020). Using artificial intelligence to detect COVID-19 and community-acquired pneumonia based on pulmonary CT: evaluation of the diagnostic accuracy. Radiology, 296(2), E65-E71.

Song, Y., Zheng, S., Li, L., Zhang, X., Zhang, X., Huang, Z., ... \& Yang, Y. (2021). Deep learning enables accurate diagnosis of novel coronavirus (COVID-19) with CT images. IEEE/ACM Transactions on Computational Biology and Bioinformatics.

He, X., Wang, S., Chu, X., Shi, S., Tang, J., Liu, X., ... \& Ding, G. (2021). Automated Model Design and Benchmarking of 3D Deep Learning Models for COVID-19 Detection with Chest CT Scans. arXiv preprint arXiv:2101.05442.

Jin, S., Wang, B., Xu, H., Luo, C., Wei, L., Zhao, W., ... \& Xu, W. (2020). AI-assisted CT imaging analysis for COVID-19 screening: Building and deploying a medical AI system in four weeks. MedRxiv.

Chen, J., Wu, L., Zhang, J., Zhang, L., Gong, D., Zhao, Y., ... \& Yu, H. (2020). Deep learning-based model for detecting 2019 novel coronavirus pneumonia on high-resolution computed tomography. Scientific reports, 10(1), 1-11.

Karakanis, S., \& Leontidis, G. (2021). Lightweight deep learning models for detecting COVID-19 from chest X-ray images. Computers in biology and medicine, 130, 104181.

Prokop, M., Van Everdingen, W.,... \& COVID-19 Standardized Reporting Working Group of the Dutch Radiological Society. (2020). CO-RADS: a categorical CT assessment scheme for patients suspected of having COVID-19 definition and evaluation. Radiology, 296(2), E97-E104. 